\title{
Conocimientos del personal de enfermería de un hospital pediátrico de referencia respecto a la evaluación y abordaje del dolor en niños
}

\author{
Martín Notejane ${ }^{1,2 *}$, Mercedes Bernadá1,2, Victoria Ruiz ${ }^{2}$ y Valentina Klisich ${ }^{2}$ \\ ${ }^{1}$ Unidad de Cuidados Paliativos Pediátricos, Departamento de Pediatría y Especialidades; ${ }^{2}$ Departamento de Pediatría y Especialidades Universidad \\ de la República, Hospital Pediátrico, Centro Hospitalario Pereira Rossell, Montevideo, Uruguay
}

\begin{abstract}
Resumen
Introducción: El dolor es un motivo de consulta frecuente, por lo que la escasa formación profesional es referida como un componente crítico para su tratamiento adecuado. El objetivo de este estudio fue describir los conocimientos y prácticas del personal de enfermería del Hospital Pediátrico del Centro Hospitalario Pereira Rossell, con respecto a evaluación y abordaje del dolor en niños. Métodos: Se llevó a cabo un estudio descriptivo, transversal. Se aplicó una encuesta anónima el 1 de diciembre de 2016 en la que se incluyó a todos los enfermeros presentes ese día. Las variables que se consideraron fueron edad, sexo, años de trabajo, nivel formativo. Para la asociación de variables se utilizó la prueba de $\chi^{2}$. Se consideró un valor significativo $p<0.05$. Resultados: La encuesta fue contestada por el $89.3 \%$ del personal presente: mediana de edad = 39 años (20-63), mujeres 81\%; mediana de tiempo como enfermero pediátrico = 10 años (1 mes-38 años); grado máximo de formación: auxiliar (73\%); nivel de conocimiento global: aceptable (61\%). No se encontró asociación significativa entre conocimiento y edad, sexo, años de trabajo y nivel formativo. Las escalas de evaluación de dolor infantil menos reportadas: neonatos, niños de 1 a 3 años y niños con trastorno cognitivo; otros déficits de conocimientos detectados: intervalo interdosis de morfina, interpretar distracción del niño como ausencia de dolor, considerar la percepción del personal de salud experimentado como la herramienta más adecuada para medir el dolor. Conclusiones: Los enfermeros encuestados demostraron conocimientos aceptables sobre evaluación y abordaje del dolor. No obstante, se objetivaron aspectos a mejorar en el conocimiento de escalas de evaluación en poblaciones especiales, falta de familiaridad con el uso de opioides y concepciones de evaluación subjetiva del dolor.
\end{abstract}

Palabras clave: Niño. Manejo del dolor. Enfermería.

\section{Knowledge of nursing staff at a pediatric referral hospital regarding the assessment and management of pain in children}

\section{Abstract}

Background: Pain is a frequent reason for consultation. Poor vocational training is referred to as a critical component for proper treatment. The objective of this study was to describe the knowledge and practices of the nursing staff of the Pedia-

Disponible en internet: 11-01-2019 Bol Med Hosp Infant Mex. 2019;76:27-34 www.bmhim.com 
tric Hospital, Pereira Rossell Hospital Center, regarding the assessment and approach of pain in children. Methods: Descriptive, cross-sectional study, anonymous survey on 1/12/2016. All the nurses present that day were included. Variables: age, gender, years of work, educational level. For the association of variables, Chi square test was used; a value of $p<0.05$ was considered significant. Results: The survey was answered by $89.3 \%$ of the personnel present; median age 39 years (20-63), women $81 \%$; median of years as pediatric nurse 10 years (1 month-38 years); maximum degree of training: auxiliary (73\%). Acceptable level of global knowledge (61\%); no significant association was found between knowledge and age, gender, years of work and educational level. Infant pain assessment scales less reported: neonates, children 1 to 3 years and children with cognitive disorder. Other deficits of knowledge detected: interval morphine interdosis, interpreting distraction of the child as absence of pain and considering the perception of the experienced health personnel as the most appropriate tool to measure pain. Conclusions: The surveys demonstrated acceptable knowledge about pain assessment and management. However, aspects to improve in the knowledge of scales of evaluation in special populations, lack of familiarity with the use of opioids and conceptions of subjective evaluation of pain were objectified.

Key words: Child. Pain management. Nursing.

\section{Introducción}

El dolor en la infancia constituye un motivo de consulta frecuente en todos los escenarios asistenciales ${ }^{1-3}$; se trata de un fenómeno complejo y multidimensional con componentes sensoriales, fisiológicos, cognitivos, afectivos, conductuales y espirituales, cuya evaluación y abordaje representa un problema de salud pública ${ }^{1-5}$. En pediatría, el dolor es frecuentemente subestimado $y$, por lo tanto, insuficientemente tratado ${ }^{6}$, lo cual refleja la imperiosa necesidad de que todos los profesionales de la salud que asisten niños cuenten con las competencias necesarias para diagnosticar y abordar el dolor de forma sistemática y adecuada ${ }^{2-5}$.

En el proceso de evaluación del dolor participan el niño, sus padres o cuidadores y los profesionales de la salud, fundamentalmente enfermeros y médicos. El personal de enfermería trabaja de forma estrecha con el paciente y su familia; son quienes más tiempo dedican al cuidado del niño y diariamente realizan procedimientos que pueden ser dolorosos, por lo que constituyen un pilar fundamental en el proceso asistencial, particularmente en la calidad de la asistencia de niños con dolor ${ }^{7,8}$. La literatura internacional refiere que el déficit de formación en la temática es una barrera importante para un adecuado abordaje de este síntoma por parte de los profesionales de enfermería ${ }^{8-10}$.

El presente estudio se llevó a cabo en el Hospital Pediátrico del Centro Hospitalario Pereira Rossell (HP-CHPR). Se trata de un hospital público de tercer nivel, centro de referencia nacional para la atención de niños y adolescentes ubicado en Montevideo, capital de Uruguay. El Hospital Pediátrico se encuentra separado del Servicio de Neonatología, ubicado en el Hospital de la Mujer. Ambos hospitales conforman el Centro Hospitalario Pereira Rossell.
El Hospital Pediátrico presenta una dotación de 140 camas en salas de internación y 20 camas de cuidados intensivos, al cuidado de 184 profesionales de enfermería. Este centro no cuenta con una política institucional para el abordaje del dolor en niños; tampoco tiene protocolizada la atención del dolor, por lo que existen diferencias en la atención por servicios (Medicina, Cirugía, Ortopedia, Cuidados Intensivos). Además, no existen consensos en el uso de escalas validadas para evaluar la intensidad del dolor ni en la forma de registro en la historia clínica de la presencia de este síntoma. Tampoco existen actividades formativas específicas brindadas por la institución.

En los últimos años, a propósito de la realización de estudios de prevalencia del dolor en niños hospitalizados en el centro ${ }^{4,5,11}$, se inició un espacio de reflexión con el objetivo de avanzar en la conformación de un equipo interdisciplinario responsable en generar protocolos de actuación en el tema.

En Uruguay existen investigaciones que evalúan el conocimiento de médicos, tanto de adultos como de niños, acerca del abordaje y tratamiento del dolor ${ }^{12,13}$. No se encontraron publicaciones nacionales respecto al conocimiento del personal de enfermería, por lo que la investigación y conocimiento del tema podría contribuir a mejorar la calidad asistencial.

El objetivo del presente estudio es describir los conocimientos y prácticas enunciadas del personal de enfermería del HP-CHPR, con respecto a la evaluación y abordaje del dolor en niños.

\section{Métodos}

Se realizó un estudio observacional, descriptivo, transversal mediante una encuesta anónima autoadministrada el 1 de diciembre de 2016. Se seleccionó una 
muestra por conveniencia. Se incluyó a todo el personal de enfermería del HP-CHPR que se encontraba presente desempeñando sus tareas en el día de la encuesta. A los profesionales que asistieron a trabajar a más de un turno, se les consultó una única vez. Se excluyeron los que no otorgaron el consentimiento y los que, por motivos personales, estuvieron ausentes ese día.

Del personal de enfermería encuestado, se describieron las siguientes variables: edad, sexo, años de trabajo como enfermero en pediatría, nivel formativo alcanzado (estudiante, auxiliar, licenciado, posgrado), sector donde desempeña tareas, asistencia a cursos y lectura de artículos vinculados al dolor en los últimos 3 años.

\section{Fuente de información}

Se realizó una encuesta mediante un cuestionario anónimo, en papel, basada en el modelo Pediatric Nurses Knowledge and Attitudes Survey Regarding Pain $(\text { PNKAS) })^{8} \mathrm{y}$ en el estudio «Valoración del dolor y aplicación de intervenciones terapéuticas enfermeras en el paciente neonatal y pediátrico, en contextos asistenciales hospitalarios" realizado en el Hospital Universitario La Paz, en Madrid 7 . El cuestionario utilizado (Anexo 1) fue adaptado a los objetivos de este estudio, sin haber sido validado previamente. Constó de 25 preguntas, 12 de tipo verdadero o falso, cuatro enunciados para indicar la opción correcta, cinco opciones con escala tipo Likert y cuatro para indicar "sí» 0 «no». El instrumento fue analizado y aprobado por dos licenciadas en enfermería con formación en cuidados paliativos y abordaje integral del dolor en niños. Para efectos de analizar la comprensión del mismo, se realizó una prueba piloto a enfermeros pediátricos que desempeñaban tareas en policlínicas del mismo centro: no fue necesario realizar modificaciones al instrumento.

El cuestionario fue entregado por los autores a cada uno de los enfermeros, y contestado por los mismos en un tiempo no mayor a media hora y sin consultar a otras personas, protocolos o materiales. Una vez completado, se separó el consentimiento informado del resto del cuestionario, colocándose en una buzonera para mantener el anonimato.

\section{Definiciones operativas}

Para efectos de este estudio, se consideró, de forma arbitraria, un nivel de conocimiento aceptable con respecto a la evaluación del abordaje del dolor en niños si el personal de enfermería encuestado respondió correctamente al menos el $60 \%$ global de las preguntas.

Las variables cualitativas se expresaron en frecuencia absoluta y relativa (\%); las variables cuantitativas, en medidas de tendencia central y su rango. Para el estudio de asociación entre variables se utilizó la prueba de $\chi^{2}$ o la prueba exacta de Fisher en los casos necesarios. En todos los casos se estableció un valor de $p \leq 0.05$ como estadísticamente significativo. Para el procesamiento de datos se utilizó el programa estadístico SPSS12.0.

\section{Consideraciones éticas}

Este estudio fue autorizado por la dirección del HPCHPR y aprobado por el comité de ética en investigación del mismo centro. Se solicitó un consentimiento informado a todos los participantes, y se salvaguardó el anonimato.

\section{Resultados}

El día de la encuesta concurrieron a trabajar al HPCHPR el $91.8 \%$ (169/184) de los enfermeros. La encuesta fue contestada por el $89.3 \%$ (151) del personal de enfermería que cumplía los criterios de inclusión. Se negaron a participar 14 funcionarios; cuatro enfermeros concurrieron a dos turnos consecutivos, por lo que se los encuestó una única vez.

La mediana de edad de los enfermeros encuestados fue de 39 años (20-63), sexo femenino $81 \%$ (122), con una mediana de tiempo de trabajo como enfermero infantil de 10 años (1 mes-38 años). Las características de la población encuestada se expresan en la tabla 1.

La media de aciertos del cuestionario en forma global fue del $61 \%$, la mediana, del $61.6 \%$, y el intervalo de confianza $95 \%$, de 58 a 65.2. No se encontró una asociación estadísticamente significativa entre los conocimientos sobre evaluación y abordaje del dolor de los enfermeros incluidos según la edad $(p=0.179)$, sexo $(p=0.123)$, años de trabajo como enfermero infantil $(p=0.305)$ o nivel de formación previa $(p=0.278)$.

En la tabla 2 se expresan las frecuencias de respuestas correctas de las preguntas de verdadero y falso.

Con respecto al conocimiento en cuanto a la selección de la escala correspondiente para evaluar el dolor según la edad o condición clínica del niño, el 66\% $(n=100)$ de los encuestados indicaron correctamente la escala numérica en escolares y adolescentes; el $45 \%(n=68)$ identificaron la escala Face, Legs, 
Tabla 1. Características de la población de enfermería encuestada del HP-CHPR $(n=151)$

\begin{tabular}{|l|c|}
\hline Variable & FR, (FA) \\
\hline $\begin{array}{l}\text { Sexo } \\
\text { Femenino } \\
\text { Masculino }\end{array}$ & $81 \%(122)$ \\
\hline Edad (años) & $19 \%(29)$ \\
\hline $20-29$ & \\
$30-39$ & $31.2 \%(47)$ \\
$40-49$ & $39.1 \%(59)$ \\
\hline Más de 50 & $17.8 \%(27)$ \\
\hline Tiempo de trabajo como enfermero infantil (años) & $11.9 \%(18)$ \\
\hline $0-5$ & \\
$6-10$ & $27 \%(41)$ \\
\hline $11-20$ & $46 \%(69)$ \\
\hline Más de 21 & $21 \%(32)$ \\
\hline Grado máximo de formación alcanzado & $6 \%(9)$ \\
\hline Auxiliar & \\
Licenciado & $73 \%(110)$ \\
\hline Posgrado & $22 \%(33)$ \\
Estudiante & $4 \%(6)$ \\
\hline
\end{tabular}

FR: frecuencia relativa; FA: frecuencia absoluta (n); HP-CHPR: Hospital Pediátrico del Centro Hospitalario Pereira Rossell.

Activity, Cry, Consolability (FLACC) para niños de 1 a 3 años; el 17\% $(n=26)$ respondieron correctamente la escala Neonatal Infant Pain Scale (NIPS) para neonatos y el $15 \%(n=23)$ identificó la escala revised-Face, Legs, Activity, Cry, Consolability (r-FLACC) para niños con trastorno cognitivo.

En cuanto al conocimiento y percepción del personal de enfermería sobre el dolor provocado por procedimientos o maniobras habituales en su práctica clínica, los encuestados consideraron que los siguientes procedimientos siempre o casi siempre generan dolor: extracción de sangre y colocación de vías venosas periféricas $(93 \%, n=141)$, colocación de sonda vesical $(80 \%, n=121)$ y aspiración de secreciones respiratorias $(62 \%, n=94)$.

Los enfermeros encuestados reportaron como imprescindible o muy importante el rol del enfermero en el alivio del dolor $(94 \%, n=143)$ y consideraron necesario el registro de dolor del paciente en la historia clínica $(97 \%, n=146)$. Con respecto a las prácticas enunciadas, el $93 \%(n=141)$ refirió realizar el registro en forma frecuente o muy frecuente, el $52.3 \%(n=79)$ reportaron lectura de al menos un artículo sobre dolor en los últimos tres años y el 38\% $(n=57)$ de asistencia a cursos o charlas sobre dolor. El $97 \%(n=146)$ de los encuestados expresaron interés en recibir cursos formativos sobre el dolor en niños.

\section{Discusión}

En el estudio que se presenta se evaluaron por primera vez, en Uruguay, los conocimientos y prácticas enunciadas de una población de enfermeros sobre la evaluación y el abordaje del dolor en niños. Los conocimientos reportados por la población encuestada fueron globalmente de nivel aceptable. Si bien la literatura internacional reporta estudios previos que indagan conocimientos y prácticas del personal de enfermería sobre esta temática, los resultados no son comparables con lo que se reporta en este estudio, dado que incluyen poblaciones heterogéneas, utilizan cuestionarios diferentes y analizan los resultados con escalas de interpretación del nivel de conocimiento distintas ${ }^{7,8,10,14}$. Independientemente de esta aclaración, la mayoría de las investigaciones reportan un déficit de conocimientos en el personal dedicado a la evaluación y abordaje del dolor en niños $7,8,14$.

En este estudio no se encontró una asociación estadísticamente significativa entre el conocimiento de los enfermeros sobre esta temática y su edad, sexo, años de trabajo como enfermero infantil o nivel formativo alcanzado. En este punto, la bibliografía consultada tampoco es concluyente. La mayoría refiere que el déficit de conocimiento detectado es generalizado, sin focalizarse en grupos de profesionales con características específicas ${ }^{8,14}$. La formación habitual del personal de enfermería contempla aspectos dirigidos a la promoción, recuperación y rehabilitación de la salud, con actitud compasiva y humanitaria. El alivio del dolor y del sufrimiento es considerado como uno de los principales derechos de los pacientes y una de las responsabilidades fundamentales del ejercicio profesional, consagradas en el Código de Ética del Comité Internacional de Enfermería9,14-16.

El nivel global del conocimiento de los enfermeros encuestados fue aceptable, aunque se detectó desconocimiento de las escalas apropiadas para evaluar dolor en niños, especialmente en los pacientes que no se pueden expresar verbalmente. La evaluación de la intensidad del dolor en niños, especialmente los menores de 5 años o que padecen algún trastorno cognitivo, es un desafío tanto para las familias como para el equipo de salud. La aplicación de escalas constituye una herramienta que contribuye a una evaluación más objetiva. Un estudio realizado en la Universidad de Dalhousie, Canadá, en 2003, indicó que el dolor en niños con trastorno cognitivo grave presentaba mayor incidencia, duración e intensidad comparado con niños sin trastorno cognitivo ${ }^{17}$. La dificultad para expresarse 
Tabla 2. Conocimientos de los profesionales de enfermería del HP-CHPR encuestados ( $\mathrm{n}=151$ )

\begin{tabular}{|l|c|c|}
\hline Enunciado & \multicolumn{2}{|c|}{ Respuestas correctas } \\
\cline { 2 - 3 } & FR (\%) & FA (n) \\
\hline Los niños pequeños tienen disminuida la sensibilidad al dolor & 69.5 & 105 \\
\hline Usar exclusivamente AINE no es efectivo para tratar dolor severo & 61 & 93 \\
\hline La escala analgésica de la OMS consta de dos escalones: AINE y AINE más opioides & 65 & 98 \\
\hline La morfina y otros opioides pueden generar adicción y dependencia & 60 & 90 \\
\hline Los analgésicos, una vez indicados, deben administrarse a intervalos regulares, no a demanda & 67 & 101 \\
\hline En un niño con dolor que recibe opioides reglados es adecuado adelantar la próxima dosis & 36 & 54 \\
\hline Las intervenciones no farmacológicas no son efectivas para el manejo del dolor & 80.1 \\
\hline Los padres no deben presenciar procedimientos dolorosos & 76.1 \\
\hline Niños menores de 8 años no pueden determinar la intensidad del dolor & 79 \\
\hline Administrar placebos no es una prueba útil para evaluar el dolor & 121 \\
\hline La percepción del personal de salud es la herramienta más adecuada para evaluar el dolor & 48.3 \\
\hline Si se logra distraer al paciente, significa que no tiene dolor severo & 36.4 \\
\hline
\end{tabular}

AINE: antiinflamatorios no esteroideos; FA: frecuencia absoluta; FR: frecuencia relativa; HP-CHPR: Hospital Pediátrico del Centro Hospitalario Pereira Rossell; OMS: Organización Mundial de la Salud.

verbalmente hace a estos pacientes más propensos a que el dolor no sea identificado por sus cuidadores y por el equipo de salud, y por ende, no sea tratado'. La separación entre el servicio neonatal ubicado en el Hospital de la Mujer y el Hospital Pediátrico donde se realizó la encuesta podría explicar la falta de adiestramiento en el uso de escalas para recién nacidos, pero también podría estar relacionado con la falta de hábito en la aplicación de escalas o de la falta de consensos acerca de qué escala usar en forma sistemática para poblaciones específicas ${ }^{5,6,10}$.

Otro déficit detectado fue el desconocimiento del intervalo interdosis de la morfina. El adelantar la dosis reglada de opioide y no administrar dosis de rescate cuando son necesarias, o no administrar la dosis correspondiente si el niño está dormido, constituyen prácticas erróneas habituales respecto al abordaje del dolor que podrían revelar desconocimiento de la cinética del fármaco. La Organización Mundial de la Salud estableció como uno de los principios para la prescripción de opioides que se administren por reloj y no a demanda, si sufre o si requiere ${ }^{1}$. La administración de morfina exclusivamente a demanda incide desfavorablemente en el control del dolor del niño, ya que es necesario que este o sus cuidadores reconozcan y expresen el dolor para que se desencadenen los mecanismos dirigidos a su control ${ }^{4}$. El desconocimiento del intervalo interdosis de la morfina también fue encontrado previamente en la encuesta realizada a residentes de pediatría $^{13}$. En ambas poblaciones, este hallazgo podría estar relacionado con la falta de familiaridad con el fármaco dada su baja prescripción en niños con dolor hospitalizados, hecho ya demostrado en publicaciones nacionales previas ${ }^{4,5,11}$.

Otras preguntas con alto porcentaje de respuestas erróneas estaban vinculadas a mitos frecuentes con respecto a la evaluación del dolor, como el uso de placebos y la distractibilidad del paciente. El efecto placebo es la modificación, a veces fisiológicamente demostrable, que se produce en el organismo como resultado del estímulo psicológico inducido por la administración de una sustancia inerte, un fármaco o un tratamiento ${ }^{17-19}$. En la práctica clínica y desde el punto de vista ético, es controversial ocultar al paciente y a su familia el uso de placebos, por lo que no deberían utilizarse $^{19-21}$.

Otro mito es que «si se logra distraer al paciente, significa que no está experimentando dolor intenso". Está demostrado que la distracción es un mecanismo de afrontamiento inconsciente hacia el dolor, lo que de ninguna manera significa que el paciente no lo esté experimentando, por lo que no es correcto utilizarlo para evaluar la intensidad del mismo $0^{4,22-24}$. 
Otra pregunta que presentó un relevante nivel de respuestas erróneas fue la que se refería a considerar «la impresión del personal de salud con experiencia como la herramienta más adecuada para la evaluación del dolor en niños». La colaboración entre padres, cuidadores y profesionales en la comprensión de las señales de dolor es fundamental en el proceso de evaluación del dolor en niños, y ha sido el sustrato para la validación de escalas objetivas para este fin. A pesar de esto, al evaluar el dolor en niños cuando este puede comunicarse, lo más relevante es su propia opinión, seguido de la de sus padres o cuidadores y, en tercer lugar, la del personal de salud que lo asiste ${ }^{23}$.

Con respecto a las prácticas enunciadas por los encuestados, la inmensa mayoría refirió realizar en forma frecuente el registro de la evaluación del dolor en la historia clínica del paciente. El tratamiento óptimo del dolor comienza con una evaluación minuciosa, la cual debe repetirse en intervalos regulares dado que la enfermedad y otros factores que influyen en ella pueden cambiar a lo largo del tiempo. Las evaluaciones periódicas permiten determinar la eficacia de las diferentes estrategias terapéuticas ${ }^{1}$. El registro de la presencia o ausencia de dolor se realiza en la historia clínica formato papel, en el apartado de registros de enfermería, ya que no existe un formulario adjunto específico para tal fin. A lo largo de su formación, los profesionales de enfermería adquieren competencias para abordar situaciones de dolor, y hacen uso de diversas herramientas para una adecuada valoración y la implementación de intervenciones terapéuticas tanto farmacológicas como no farmacológicas. La North American Nursing Diagnosis Association (NANDA) menciona dos etiquetas diagnósticas relativas al dolor (dolor agudo y dolor crónico ${ }^{25}$. EI HP-CHPR se encuentra en un proceso de informatización de la historia clínica de los usuarios asistidos a nivel ambulatorio y durante la hospitalización. Además, en el apartado de registros de enfermería, incorpora artículos sobre la evaluación periódica del dolor, uso de escalas validadas e intervenciones farmacológicas y no farmacológicas para su tratamiento. Estas podrían ser estrategias que contribuyan a protocolizar la actuación de los profesionales en el marco de una política institucional para el abordaje del dolor de niños y adolescentes.

Como limitaciones de este estudio se destaca el hecho de no haber realizado un cálculo muestral, incurriendo en un sesgo de selección. Si bien la mayoría de los enfermeros presentes el día de la encuesta participaron, no es posible generalizar los resultados a la totalidad de los enfermeros pediátricos del centro.
Otra limitación fue la utilización de un cuestionario no validado, basado en dos instrumentos previos adecuadamente validados. Estas modificaciones pudieron alterar la capacidad del instrumento para detectar el nivel de los conocimientos de los enfermeros en esta temática. Como fortaleza, este es el primer estudio en Uruguay que explora los conocimientos y prácticas enunciadas por enfermeros pediátricos sobre la evaluación y abordaje del dolor en niños. La importante participación de los profesionales de enfermería fue vital para lograr resultados válidos para la población encuestada. Sería oportuno replicar esta investigación utilizando un cuestionario validado y realizando previamente un cálculo muestral después de establecer protocolos institucionales de actuación sobre el abordaje y evaluación del dolor en niños.

El personal de enfermería encuestado demostró conocimientos aceptables sobre la evaluación y el abordaje del dolor en niños. No obstante, se objetivaron carencias en el conocimiento de las escalas de evaluación del dolor en poblaciones específicas y falta de familiaridad con el uso de opiáceos. Además, se constataron dificultades en la evaluación y algunos mitos que apoyan la necesidad, reconocida por el propio personal encuestado, de formación continua en la temática.

Es probable que las debilidades metodológicas relacionadas con la selección de una muestra por conveniencia y el uso de un cuestionario no validado pudieran influir en los resultados encontrados.

\section{Responsabilidades éticas}

Protección de personas y animales. Los autores declaran que para esta investigación no se han realizado experimentos en seres humanos ni en animales.

Confidencialidad de los datos. Los autores declaran que han seguido los protocolos de su centro de trabajo sobre la publicación de datos de pacientes.

Derecho a la privacidad y consentimiento informado. Los autores declaran que en este artículo no aparecen datos de pacientes.

\section{Conflicto de intereses}

Los autores declaran no tener ningún conflicto de interés.

\section{Financiamiento}

No se contó con financiamiento para esta investigación. 


\section{Bibliografía}

1. Organización Mundial de la Salud. Directrices de la OMS sobre el tratamiento farmacológico del dolor persistente en niños con enfermedades médicas. Ginebra: AVENUE APPIA; 2012. Disponible en: http://www who.int/medicines/areas/quality safety/3PedPainGLs coverspanish.pdf

2. Stevens B, Harrison D, Rashotte J, Yamada J, Abbott L, Coburn G, et al. Pain assessment and intensity in hospitalized children in Canada. J Pain. 2012;13:857-65.

3. Friedrichsdorf S, Postier A, Eull D, Weidner Ch, Foster L, Gilbert M, et al. Pain outcomes in US Children's Hospital: a prospective cross sectional survey. Hosp Pediatr. 2015;5:18-26.

4. Moraes M, Zunino C, Virginia D, Ponte C, Favaro V, Bentancor S, et al. Evaluación de dolor en niños hospitalizados en servicios de salud públicos y privados de Uruguay. Arch Pediatr Urug. 2016;87:198-209.

5. Zunino C, Notejane M, Bernadá M, Rodríguez L, Vanoli N, Rojas M, et al. Dolor en niños y adolescentes hospitalizados en un centro de referencia. Rev Chil Pediatr. 2018;89:67-73.

6. Montes A, Arbonés E, Planas J, Muñoz E, Casamitjana M. Los profesionales sanitarios ante el dolor: estudio transversal sobre la información, la evaluación y el tratamiento. Rev Soc Esp Dolor. 2008;2:75-82.

7. Sellan M, Díaz M. Valoración del dolor y aplicación de intervenciones terapéuticas enfermeras en el paciente neonatal y pediátrico, en contextos asistenciales hospitalarios. Rev Cubana Enfermer. 2012;28:144-55.

8. Lobete C, Rey C, Kiza A. Comparación de los conocimientos sobre el dolor infantil en dos poblaciones de profesionales de enfermería. An Pediatr (Barc). 2015;82:e158-64.

9. Rodriguez I, Fajardo A, Peña M. Rol del enfermero en las etapas de evaluación valoración e intervención del dolor en niños. Rev Haban Cienc Med. 2016;15:399-407.

10. Merino D, García F, Mariscal M, García M, López M, Rozas G. Manejo del dolor infantil por los profesionales de enfermería. Evidentia. 2008;5.

11. Cristiani $F$, Hernández A, Sálice L, Orrego $P$, Aráujo M, Olivera L, et al Prevalencia de dolor en niños hospitalizados en el Centro Hospitalario Pereira Rossell. Anest Analg Reanim. 2013;26:3-16.
12. Píriz G, Estragó V, Pattarino $C$, Sandar T. Dolor oncológico: un problema no resuelto. Encuesta a médicos residentes del Hospital Maciel. Rev Med Urug. 2004;20:32-43.

13. Notejane M, Le Pera V, Bernadá M. Conocimientos relativos al abordaje del dolor en niños. Encuesta a postgrados y residentes. Arch Pediatr Urug. 2016;87:323-31.

14. Aguilar M, Mur N, Padilla C, García Y, García R. Actitud de enfermería ante el dolor infantil y su relación con la formación continua. Nutr Hosp. 2012;27:2066-73.

15. Salantera S, Lauri S. Nursing students' knowledge of and views about children in pain. Nurse Educ Today. 2000;20:537-47.

16. Anderson T. Pain management nursing: scope and standars of practice. Nebr Nurse. 2011;43:8-9.

17. Breau L, Camfield $C, M c G$ rath $P$, Finley $G$. The incidence of pain in children with severe cognitive impairment. Arch Pediatr Adolesc Med. 2003;157:1219-26.

18. Shapiro AK. Factors contribuing to the placebo effect. Their implications for psychotherapy. Am J Psychother. 1964;18:73-88.

19. Lam R, Hernández P. El placebo y el efecto placebo. Rev Cubana Hematol Inmunol Hemoter. 2014;30:214-22.

20. Rodriguez-Raecke R, Niemeier A, Ihle K, Ruether W, May A. Structural brain changes in chronic pain reflect probably neither damage nor atrophy. PLoS One. 2013;8:e54475.

21. Scublinsky D. El efecto placebo y el dolor. Rev Arg Reumatol. 2014;25:06.

22. Friedrichsdorf S. Multimodal pediatric pain management (Part 2). Pain Manag. 2017;7:161-6.

23. Schechter N, Finley A, Bright N, Laycock M, Forgeron P. Childkind: a global initiative to reduce pain in children. Pediatr Pain Letter. 2010;12:26-30

24. Oberlander T, Craig K. Pain and Children with Developmental Disabilities. En: Schechter N, Berde C, Yaster M. Pain in infants, children, and adolescents. Philadelphia: Lippincott Williams and Wilkins; 2006. pp. 599-619.

25. Santamaría JM, Arribas A, Sellán MC, Vázquez A, Díaz ML, Amézcua A. Limitaciones en el cuidado debidas a la construcción de los diagnósticos del dolor. Lenguaje del Cuidado. 2010;2:33-4. 


\section{ANEXO 1. Formulario de encuesta}

\section{Completar con cruz, círculo, letra o número según corresponda.}

Datos patronímicos:

Género Edad Años de trabajo como enfermero infantil

Grado de formación alcanzado:

Estudiante....

Licenciado....

Auxiliar....

Posgrado.......

Referente a la evaluación y abordaje del dolor en niños, señale verdadero $(V)$ o falso $(F)$ :

1) Los niños pequeños tienen un sistema neurológico en desarrollo y por eso tienen disminuida la sensibilidad al dolor. (Falso)

2) Usar exclusivamente ibuprofeno u otros antiinflamatorios no esteroideos (AINE) no es efectivo para el tratamiento del dolor severo. (Verdadero)

3) La escala analgésica de la Organización Mundial de la Salud (OMS) consta de dos escalones: uno de AINE y otro de AINE más opioides. (Verdadero)

4) La morfina y otros opioides se caracterizan por generar adicción y dependencia, por lo cual no es aconsejable su uso en pediatría. (Falso)

5) Los analgésicos, una vez indicados, deben administrarse a intervalos regulares y no únicamente cuando el paciente tiene dolor (a demanda). (Verdadero)

6) Usted está asistiendo en sala a un niño cursando postoperatorio de cirugía; el mismo recibe analgesia con morfina cada 4 horas por vía intravenosa, y tiene indicado hasta tres rescates si lo requiere. Cuando llega a valorarlo, el paciente se encuentra dolorido. Dado que recibió la última dosis reglada hace 3 horas, es adecuado adelantar la próxima dosis. (Falso)

7) Las intervenciones no farmacológicas no son efectivas para el manejo del dolor. (Falso)

8) Los padres no deben estar presentes durante los procedimientos dolorosos. (Falso)

9) Los niños menores de 8 años no son capaces de determinar la intensidad del dolor y por eso el enfermero debe basarse en la evaluación que hacen los padres. (Falso)

10) Administrar a los pacientes placebos no es una prueba útil para determinar si el dolor es real. (Verdadero)

11) La percepción del personal de salud experiente es la herramienta más adecuada para medir el dolor en niños. (Falso)

12) Si se logra distraer al paciente, significa que no está experimentando dolor intenso. (Falso)
Pacientes con dolor, aplicación de escalas de evaluación de intensidad.

13) Indique la escala correcta para evaluación del dolor en recién nacidos:
a) Escala de caras
b) Numérica (EVA)
c) r-FLACC
d) NIPS (correcta)
e) FLACC

14) Indique la escala correcta para evaluación del dolor en niños menores de 3 años:
a) Escala de caras
b) Numérica (EVA)
c) $r-F L A C C$
d) NIPS
e) FLACC (correcta)

15) Indique la escala más utilizada para evaluación del dolor en escolares y adolescentes:
a) Escala de caras, b) Numérica (EVA) (correcta)
c) $r$-FLACC
d) NIPS
e) FLACC

16) Indique la escala correcta para evaluación del dolor en pacientes con trastorno cognitivo en mayores de 3 años:
a) Escala de caras
b) Numérica (EVA)
c) r-FLACC (correcta)
d) NIPS
e) FLACC

Con respecto a las siguientes maniobras, indique con qué frecuencia pueden producir dolor:
a) Nunca
b) Casi nunca
c) Casi siempre
d) Siempre

17) La extracción de sangre o colocación de vía venosa periférica (siempre o casi siempre)

18) La colocación de sonda vesical (siempre o casi siempre)

19) La aspiración de secreciones respiratorias (siempre o casi siempre)

Con respecto al rol del enfermero frente al dolor:

20) El rol del enfermero en el alivio del dolor es (marque una opción):
a) Ninguno
b) Poco importante
c) Muy importante
d) Imprescindible

21) ¿Considera necesario el registro del dolor del paciente en la historia clínica por parte de enfermería? Sí/NO

22) ¿Con qué frecuencia realiza el registro de la evaluación del dolor?
a) Nunca
b) Casi nunca
c) Frecuentemente
d) Muy frecuentemente

23) ¿Ha leído algún/os artículo/s o revista/s sobre dolor en los últimos 3 años? Sí/NO

24) ¿Ha asistido a charlas, cursos o actualizaciones sobre dolor en los últimos 3 años? Sí/NO

25) ¿Le interesaría recibir cursos o talleres de formación específica sobre abordaje del dolor en Pediatría? Sí/NO 